Andrzej Bonasewicz

\title{
LAS MIGRACIONES DE LA POBLACIÓN Y DESARROLLO REGIONAL EN AMERICA LATINA
}

El desarollo regional de los paises en vias de desarollo est determinado pố la internacional división de trabajo y la especialidad funcional. Este desarrollo es condicionado tanto por el potencial economico y dempgrafico como por los capitales de las entidades territoriales. La desigualidad de la organizacion espacial de los respectivos territorios es la consecuencia de sste fenomeno. Un papel muy importante juegan aqui los factores demograficos: el número de los habitantes, su reproduccion y la estructura demografica tanto de los asentamientos como las migraciones de la poblacion.. Los mencionados factores por un lado condicionan el desarrollo regional y por el otro, son sus consecuencias.

Las migraciones de la población juegan el papel especiai; su intensificacion y las direcciones estan relacionadas con el desarrollo regional. Muy a menudọ se dice que desempeñan ,el papel de tornasol" de la situación socioeconómica. Las inmigraciones se dirigen especialmente hacia las regiones más atractivas, con las mejores condiciones de vida, altas rentas per capita y las mejores condiciones de trabajo. Los emigrantes provienen sobre todo de los terrenos con la presión demografica, con alta o baja densidad de la población, los cuales no tienen suficientes determinantes del desarrollo regional.

En América Latina las migraciones de la población tienen las tradiciones historicas y en el curso de su historia siempre senalaban las direcciones del desarrollo regional. Esto empezo cuando la conquista y seguia durante la colonización europea despues de la independencia de América Lataina. Un papel especial juegan las migraciones internas hacia las regiones centrales y las periféricas, abudantes en los recursos naturales, denominadas , las regiones de nuevas posibilidades". Los emigrantes provienen de las regiones periféricas, las cuales no tienen las posibilidades del desarrollo y que son denominadas como depresivas y neutrales. Cada a no la movi lidad de la población aumenta y cada vez con más intensidad los habitantes de América Latina cambian las entidades administrativas de su nacimiento. 
Los datos de los censos at la población informan sobre los habitantes naciódos en enídades admmistrativas de la primera jerarquia y residentes en otras. Estos datos permiten obtene: la infornación sobre emumero de las personas, que han cambiado las entidades aúministrativas de la primera jerarquia de su nacimiento. En 1950 cerea de $13 \%$ de los habitanties de America Latina han cambiado las entidades de su nacimiento. mientras que en 1971 más de $25 \%$ Los datos del 1980 demuestran, que el porciento de migrantes in: srecito, to que significa el dinámico desarrollo de algunos territorios y la profuncización de las desigualidades del desarrollo.

Para demostrar fenomenos de las migraciones en America Latina se ha becho un analisis mas detallado de los flujos migratorios en tres paises, muy ripicos para, el continente: Mixico, Venezuela y Brasil durants los años de los censos 1954 y 1980, usando los indices de los saldos migratorios y el coeficiente de efecrividad de las migraciones. El coeficiente de electividad, te las migraciones se calcula por la formula:

donde: $I$ inmigración

$$
C^{\prime}=\frac{I-E}{I+E} \cdot 100
$$

$E$-. emigración.

Este coeficienie se cambia entrc $+100 y-100$. Cuando es mayor del $O$, el salite migratorio es pesitivo, cuando menor, es negativo. Según este coeficiente podemos analizar el valor relativo de los flujos migratorios.

Mixico pertenece a los paises con alta tasa de crecimiento de la población. En 1950 tenia 25,8 miliones de habitantes, mientras que en 1980 ya 67.4 milliones. Las migraciones internas en 1950 contenian 3288,1 mil personas $(12.7 \%$ de la poblacion total). aumentaron en 1980 a las 7254,6 mil personas ( $10.8 \%$ de la poblacion total).

En 1950 casi la mitad de los inmigrantes $(48,7 \%$ ) llegaba al Distrito Federal, es decir a la ciudad de Mexico. Todos los estados cercanos al Distrito Federal tenian el saldo migratorio negativo, Unos altos coeficientes de la efectividad de las migraciones tenian tambien los estados fronterizos con Estados Unidos y las zonas poco pobladas como Baja California Sur, Quintana Roo y el estado petrolifero Tamaulipas. Los estados de la parte central y meridional fueron emigratorios. El mayor numero de los emigrantes provenia de lós estados de México, Jalisco. Guanajuato y Michoacan $(34,7 \%)$. Ni siquiera grandes ciudades como Guadalajara o Acapulco, podian disminuir el flujo de los emigrantes de Jalisco y Guerrero. Las causas de este fenomeno hay que buscarlas en el gran centralismo de la actividad industrial en zona metropolitana de Mexico, como en las mejores condiciones de trabajo en la zona fronteriza con Estados Unidos.

En 1980 cambiaron las direcciones y la intensidad de las migraciones internas. Disminuyó atractivo del Distrito Federal donde inmigraron 20,6\% de todos los inmigrantes. El segundo lugar ocupaba el estado de Mexico con 


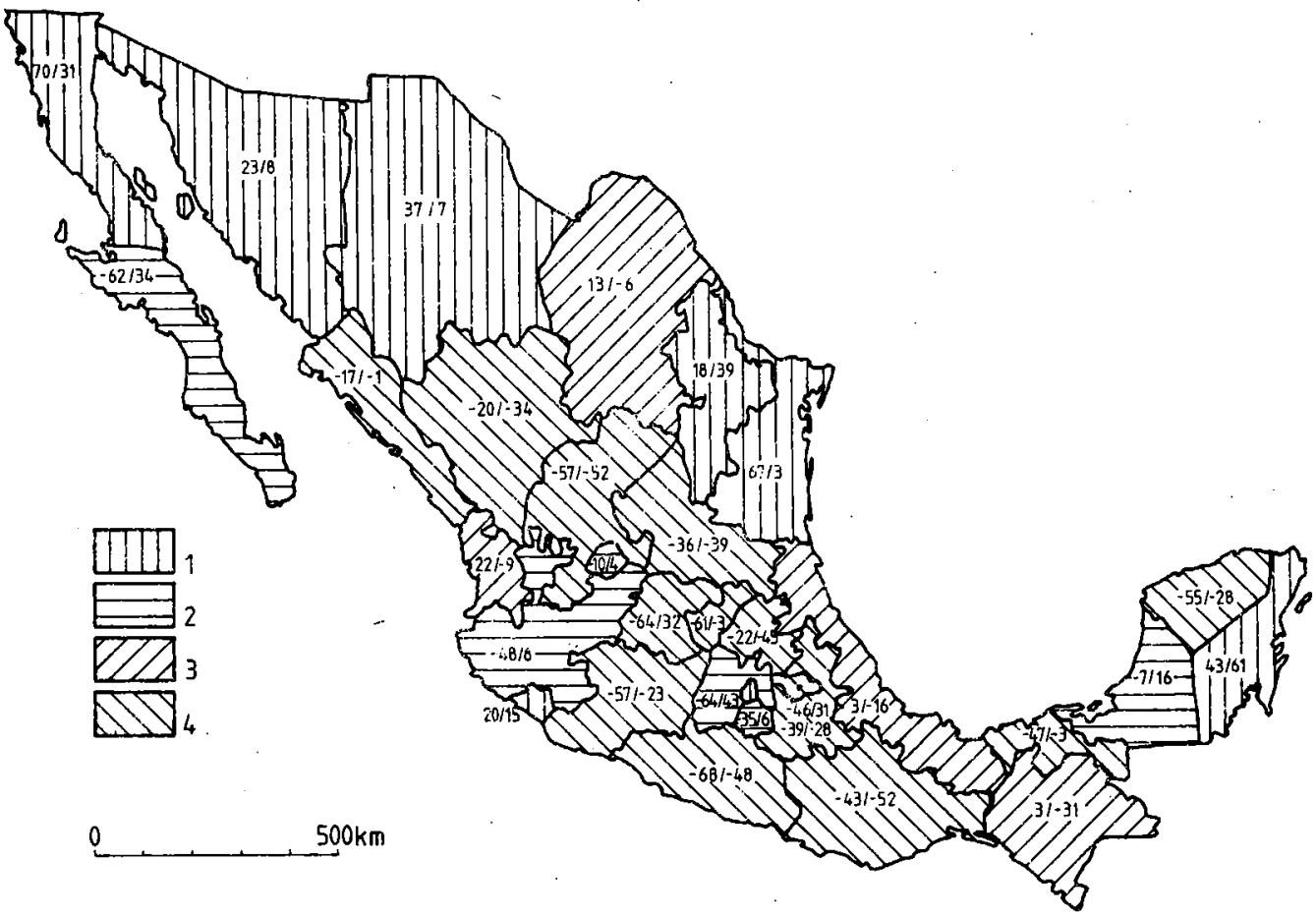

Mapa N 1. Mexico. Coeficience de efectividad de las migraciones en 1950 y 1980. Valor del coeficiente (cifra izquierda 1950. derecha 1980): 1. positivo en 1950, positivo en 1980. 2. negativo en 1950 , positivo en 1980,3 . positivo en 1950 . negativo en 1980. 4. negativo en 1950, negativo en 1980

$19,8 \%$ inmigrantes. La causa de este fenómeno fue relacionada con el desarrollo de la zona industrial en estado de Míxico y la deglomeración de la zona metropolitana. En tercer lugar se encortraron los estados con mayores ciudades-Jalisco con Guadalajara y Nuevo Leon con Monterrey, que abarcaban el $11 \%$ de los inmigrantes. Disminuyó el atractivo de la zona fronteriza con Estados Unidos. Un alto coeficiente de la efectividad de las migraciones tenia el territorio de Quintana Roo, la zona de nueva colonizacion en la peninsula de Yucatan. La emigracion maxima venia del Distrito Federal, de donde provenia el $7,2 \%$ de los emigrantes, del estado de Mexico 5,9\% y Veracruz 5,9\%. Los mayores coeficientes negativos tenian los estados Zacatecas y Oaxaca. Disminuyo bastante la inmigración a los estados Baja California Norte y Baja California Sur como a la zona petrolifera de Tamaulipas, mientras que aperecio la trueva zona petrolifera Campeche, con un saldo postivo de migraciones.

Comparando los valores del coeficiente de la efectividad de las migraciones en 1950 y 1980 se puede concluir, que disminuyeron las diferencias entre los valores maximos; y minimos; decir disminuyeron las migraciones interestadales, en cifras relativas. Esto significa una politica 
regional mas equilibrada dentro del pais. El aumento de los inmigrantes en zonas vecinas de la capital, que es una prueba de la deglomeración de las zonas fronterizas, poco pobladas resultan también de la politica regional.

Venezuela, un pais con gran dinamica del desarollo después de la segunda guerra mundial, se caracterizaba por la gran participacion de las migraciónes internas. También la inmigración extranjera tenia un papel significante. La población aumento desde 5035 mil en 1950 hastá 14517 mil personas en 1981 , casi 3 veces durante los 31 años. La estructura espacial tanto demografica como económica demuestra grandes desigualidades. La región central, que ocupa 2,34\% del territorio nacional (Distrito Federal y los estados Aragua, Carabobo y Miranda) fue poblada en 1950 por $26 \%$ de los habitantes, mientras que en 1981 por el $37,5 \%$ de la población nacional. El $18,8 \%$ de los habitiantes de Venezuela en 1950 cambió la entidad ad-

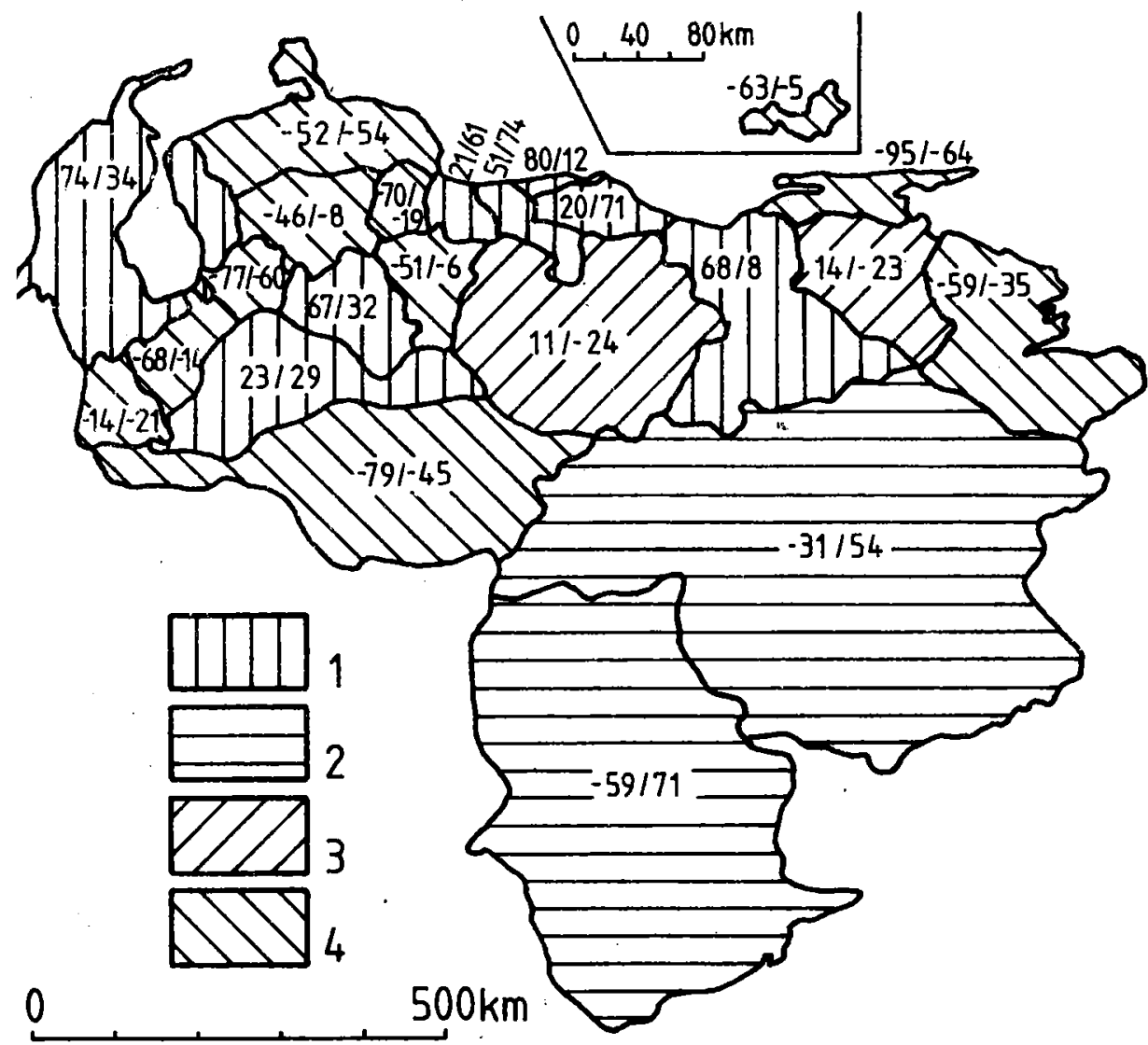

Mapa N 2. Venezuela. Coeficiente de efectividad de las migraciones en 1950 y 1981. Valor del coeficiente (cifra izquierda 1950, derecha 1981): 1. positivo en 1950, positivo en 1981. 2. negativo en 1950, positivo en 1950, negativo en 1981,4 . negativo en 1950 , negativo en 1981 
ministrativa de su nacimiento, mientras que en 1981 el 28,7\%. Eso quiere decir, que las migraciones internas aumetaron bastante, no solo en los numeros absolutos, pero también en los relativos.

En 1950 là mayoria de los inmigrantes se dirigia al Distrito Federal, vecino estado Aragua y a los dos estados petroliferos Zulia Anzoategui (en total $87,5 \%$ de los inmigrantes internos y externos). El saldo positivo de migraciones tenian tambien las zonas de colonizacion agricola: Barinas y Portuguesa, estados Ilaneros Guarico y Monagas y los estados de la región central Carabobo y Miranda. Los restandes entidades administrativas tenian el saldo negativo. El mayor número e los emigrantes provenia de los estados Surce $(22,2 \%)$ y Trujillo $(20,1 \%)$. El atractivo de la parte septentrional de Venezuela para los inmigrantes fue relacionado con la politica de substitucion de las importaciones, la cual favorecia la región central, ubicada en la costa maritima, vecina a los estados extranjeros.

En 1981 la situacion cambió de nuevo. El stractivo del Distrito Federal perdio su importancia a favor de los estados vecinos: Aragua y Carabobo y especialmente de Miranda. A la región central se dirigia el $57,7 \%$ de los todos inmigrantes extrannjeros y también la mayoria de los nacionales (el 51,6\%). Los altos coeficientes de la efectividad de las migraciones (salvo los estados de la region central), tenian el estado Bolivar y territorio Amazonas, lo que fue relacionado con el desarrollo del polo desarollo en Guayana, con la explotación y transformación del mineral de hierro. Disminuyeron los coeficientes en las zonas petroliferas de Zulia y Anzoategui y de la colonización agricola de Portuguesa y Barinas. Todas otras entidades administratives tenian un saldo migratorio negativo. La mayoria de los emigrantes provenia de Falcón, Sucre y Trujillo. El Distrito Federal tenia un saldo positivo, pero solo gracias a la inmigración extranjera. También el estado de Tachira, zona de gran emigración, tenia un pequeño saldo negativo gracias a gran inmigración de los colombianos.

Comparando los datós de 1950 y 1981 se puede decir, que en alguanas zonas disminuyo la emigración relativa (sobre todo en Nueva Esparta) gracias a la politica del comercio libre y también en las zonas de agricultura intensiva y de industria alimenticia (en Lara, Yaracuy y Cojedes). La inmigración aumentó basante en Bolivar, mientras que disminuyó en Zulia y Anzoategui (las zonás petroliferas) y surgió un saldo negativo en Monagas y Guarico. Los coeficientes de la efectividad de las migraciones tanto positivos como negativos en 1981 son menores que en 1950 , lo que significa las migraciones más equilibradas.

Brasil, el mayor pais de América Latina en el cual durante los 30 años (1950-1980) aumento el numero de los habitantes de 51976 mil a 119071 mil. La poblacion esta distribuida de un modo muy desigual. En 1950 en el $65 \%$ de la superficie total del pais, vivia solamente $7 \%$ de los habitantes del pais, mientras que en el $18 \%$ de la superfice nacional vivia $69 \%$ habitantes de Brasil. En 1980 en el $65 \%$ 


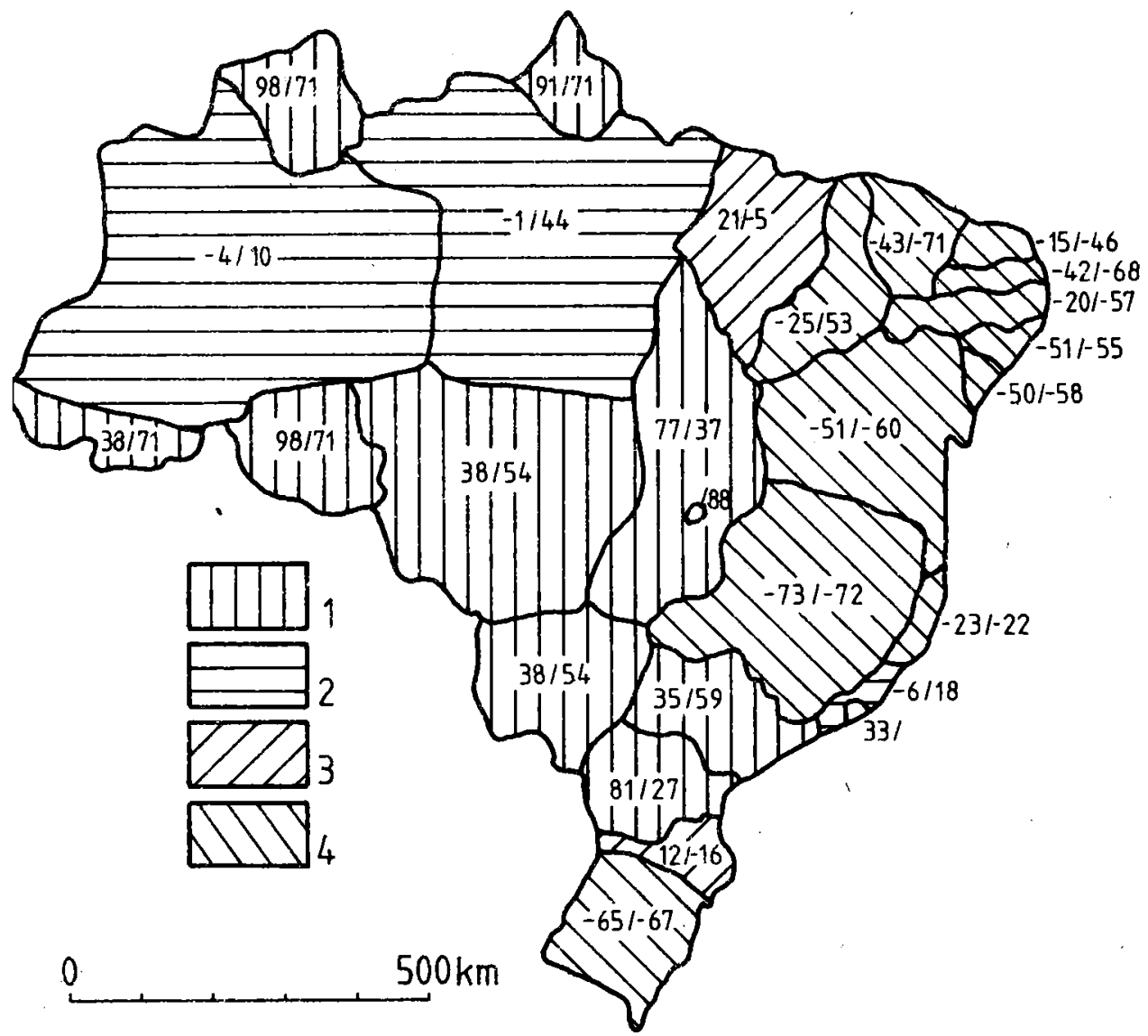

Mapa N 3. Brasil. Coeficiente de efectividad de las migraciones en 19: y 1980. Valor dei coeficiente (cifra izquierda 1950, derecha 1980): 1. positivo en 1950, positivo en 1980. 2. negativo en 1950, positivo en 1980. 3. positivo en 1950, negativo en 1980. 4. negativo en 1950 , negativo en 1980

de esta superfice ya vivia un $12 \%$ de los habitantes, mientras que en el $18 \%$ de ella vivia el $72 \%$ de los brasilenos. Estas cifras indican, que en Brasil existen los movimientos migratorios de la población. Por un ládo existe una tendencia hacia la mayor concentración espacial y por otra la colonización de los territorios poco poblados. Las migraciones interestadales en 1950 llegaron consigo cerca del 10\% de los habitantes, mientras que en 1980 ya el $15 \%$ y tienen una tendencia creciente.

$E^{\prime}$ analisis de las migraciones en 1950 indica, que para los inmigrantes los estados mas atractivos fueron: Sao Paulo con $20,9 \%$ de todos los inmigrantes, Guanabara (es decir ciudad Rio de Janeiro) con 18,3\% y Parana con el $13 \%$. Esta inmigracion se dirigia principalmente a las ciudades mas importantes como Sao Paulo y Rio de Janeiro. En Parana fue relacionada con la colonizacion agricola en parte septentrional de este estado. 
Analizando el origen de los emigrantes podemes decir. que el $26,3 \%$ de ellos provenia de Minas Gerais, el 9.8\% de Sao Paulo, el $9.7 \%$ de Rio de Janeiro y el $8,5 \%$ de Bahia. Los coeficientes positivos de la efectividad de las migraciones tenian los estados orientales y centro-occidentales, mientreas que negativos tenian los estados de la parte septentrional y nororiental. Solamente los territorios federales, ubicados en las zonas fronterizas tenian el saldo positivo.

En el 1980 la situación cambio. Aumentó bastante la emigración de la parte nororiental y meridional, mientras que los estados y teritorios de la parte septentrional y centro-occidental tenian el saldo positivo, como efecto de la politica "marcha para occidente". Sobre todo las zonas fronterizas (los territorios federales) tenian un alto valor del coeficiente de la efectividad de las migraciones. La mayoria de los inmigrantes llegaba a los estados Såo Paulo (el 32,1\%), Rio de Janerio (el 14,2\%), Paraná (el : $1: 7 \%$ ), Goiás (el 32.1\%), Distrito Federal (el 4,4\%). Estas cifras indican el gran atractivo de la region central de Brasil, formando por las zonas vecinas de los ciudades mas grandes del pais como Sa Paulo y Rio de Janeiro. El atractivo de Paraná ha disminuido por las malas cosechas, causadas por los desastres climaticos. El atractivo de Goias y Distrito Federal fue relacionado con la creación de la capital nacional en Brasilia y el aumento de la actividad industrial en Goiás. El analisis de la emigracion demuestra, que el 21,6\% de los emigrantes nacionales provenia de Minas Gerais, el $10,5 \%$ de Bahia, el $8,3 \%$ de Sao Paulo, el $6,7 \%$ de Parana, el $5.2 \%$ de Ceara, el $5,2 \%$ de Pernambuco y el $5,1 \%$ de Rio Grande do Sul. Los coeficientes de la efectividad de las migraciones, en mayoria de los casos aumentaron especialmente en la región nororiental, tanto en numeros absolutos como en relativos. También los estados meridionales teisian el saldo negativo: Rio Grande do Sul y Santa Catarina. El saldo positivo aumentó especialmente en Sào Paulo. Las existentes tendencias demuestran. que el desarollo socioeconomico de Brasil es muy polarizado y las discrepancias territoriales en comparación con el 1950, se, profundizaron. El aumento de la significación de las grandes ciudades es muy tipica para Brasil, la cual tiente mas que 12 ciudades millionaris en su territorio. En los 30 anos investigados aumentaron las diferencias entre los mayores y menores valores del coeficiente de la efectividad de las migraciones, lo que un desequilibrado desarrollo socioeconómico del pais.

La comparación de los movimientos migratorios de la población en estos iies paises permite establecer unas semejanzas. En los anos cincuenta la mayoria de los inmigrantes se dirigia a las capitales mientras que en los anos ochenta a las zonas proximas de las capitales y los estados limitrofos. Este hecho fue relacionado con unas pruebas de la deglomeración de las capitales y con las mejores posibilidades de las inversiones en la cercania de las zonas metropolitanas. El aumónto de las inmigraciones a las entidades con grandes ciudades significa, que la inmigracion se dirige principalmente 
a las grandes ciudades. El alto coeficiente de la efectividad de las migraciones en las zonas poco pobladas significa una politica de la colonizacion planificada, la creacion de unas zonas atractivas para los inmigrantes como Bolivar en Venezuela, Quintana Roo y Baja California en Mexico, los territorios federales en Brasil. Las zonas de exploatación petrolera pueden ser inmigratorias o emigratorias,, dependen de las tendencias en produccion, como Tamauliapas, Veracruz o Campeche en Mexico, Zulia, Anzoategui o Monagas en Venezuela. Los territorios de la colonización agricola son atractivos para los inmigrantes como Baja California Norte en México, Portuguesa y Barinas en Venezuela, Rondonia y Parana en Brasil. Las grandes ciudades en las zonas de emigración, aparte de estas, que reciben los inmigrantes, no pueden frenar el movimiento emigratorio como Acapulco, Veracruz y Mérida en México, Barquisimeto, San Cristobal y Mérida en Venezuela, Recife, Salvador, Belo Horizonte, y Porto Alegre en Brasil.

El analisis de los movimientos migratorios de la poblacion es muy importante para las investigaciones sobre desarrollo regional. Los cambios de las tendencias y direcciones de los movimientos de la poblacion, significan los cambios en el desarrollo regional. El conocimiento de este fenomeno permite planificar mejor el desarrollo. 